\title{
The functional anatomy of the frontal lobes
}

\section{Parashkev Nachev, Christopher Kennard and Masud Husain}

In their illuminating recent article (Is the rostro-caudal axis of the frontal lobe hierarchical? Nature Rev. Neurosci. 10, 659-669 $(2009))^{1}$, Badre and D'Esposito generalize to the frontal lobes as a whole a point we recently made about the medial frontal cor$t^{2}$ : that the functional architecture suggests a continuous rostro-caudal gradient reflecting the conditional complexity of the associated behaviour. Reviewing a broad swathe of behavioural and neurophysiological studies, they argue - convincingly, in our view that a wealth of data now supports this new conceptual framework for frontal lobe function. However, there are some important consequences of such a perspective that might not be apparent at first glance and that merit some further consideration.

First, if the gradient model is correct, traditional discrete models of frontal lobe functions being compartmentalized to highly demarcated zones cannot be. Not only do the empirical data support the former, they also strongly undermine the latter. The frontal lobes can have only one functional architecture. As most models of frontal lobe function - indeed of brain function generally - are constructed on the basis of more or less discrete functional units, if gradient models are correct then a re-evaluation of such models in cognitive neuroscience is required. To confirm or disprove such a perspective is therefore a task of the highest priority.
Second, current methodological tools are peculiarly ill-suited to the study of gradient models and to distinguishing between discrete and continuous functional architectures in the brain. This is partly so because sampling is either microscopic (for example, single-cell recordings) or macroscopic (for example, functional imaging and lesion studies). The intermediate, 'mesoscopic', scale at which a gradient organization is likely to be embedded therefore falls within a methodological blind spot. Furthermore, as noise is typically continuous, it is always technically easier to find support for a discrete model than for a continuous one, whatever the underlying reality. The task is thus not only important but extremely difficult.

Third, if the gradient is indeed rostrocaudal, what are the functional differences between regions that lie on the same point on that gradient, but on lateral versus medial frontal surfaces? One way to answer this question is to sample at a small scale (for example, by making single-unit recordings) but to cover large areas of cortex, probing activity on the same behavioural task in awake, behaving primates. Unfortunately, this has very rarely been performed - for understandable reasons. However, one exception is the elegant series of studies performed by Hoshi and Tanji on both lateral and medial frontal regions ${ }^{3,4}$.
Although the results of these studies are separated in different papers, a review of the results demonstrates some differences but also many strong similarities between the pre-supplementary motor area, the supplementary motor area and the lateral premotor cortex. Thus, medial and lateral frontal areas may not be as discrete as is sometimes envisaged.

Finally, many well-established conceptual devices cease to have any meaning within a gradient model framework. Although one can have a continuous hierarchy - or at least a hierarchy that is discretized at a mesoscopic scale - it no longer makes any sense, for example, to speak of the binary labels of supervisor and supervised ${ }^{5}$. If the gradient perspective is right, the very language of cognitive neuroscience may have to change.

Parashkev Nachev and Masud Husain are at the Institute of Cognitive Neuroscience and the Institute of Neurology, University College London, Alexandra House, 17 Queen Square, London, WC1N 3AR, UK.

Christopher Kennard is at the Department of Clinical Neuroscience, Imperial College London, St Dunstan's Road, London, W6 8RP, UK.

Correspondence to M.H. e-mail:m.husain@ion.ucl.ac.uk doi: 10.1038/nrn2667-c1 Published online 14 October 2009

1. Badre, D. \& D'Esposito, M. Is the rostro-caudal axis of the frontal lobe hierarchical? Nature Rev. Neurosci. 10, 659-669 (2009).

2. Nachev, P., Kennard, C. \& Husain, M. Functional role of the supplementary and pre-supplementary motor areas. Nature Rev. Neurosci. 9, 856-869 (2008).

3. Hoshi, E. \& Tanji, J. Contrasting neuronal activity in the dorsal and ventral premotor areas during preparation to reach. J. Neurophysiol. 87, 1123 (2002)

4. Hoshi, E. \& Tanji, J. Differential roles of neuronal activity in the supplementary and presupplementary motor areas: from information retrieval to motor planning and execution. J. Neurophysiol. 92 , 3482-3499 (2004).

5. Norman, D. A. $₫$ Shallice, T. in Cognitive Neuroscience: A Reader (ed. Gazzaniga, M. S.) 376-390 (Wiley, 2000). 